Homology, Homotopy and Applications, vol.5(1), 2003, pp.213-218

\title{
P-EMBEDDINGS, AR AND ANR SPACES
}

\author{
L. STRAMACCIA \\ (communicated by Walter Tholen)
}

\begin{abstract}
Generalizations of Borsuk's characterization theorems for AR and ANR spaces (for metrizable spaces) are provided. Moreover, a simple proof of Morita's theorem is given, in generalization of Borsuk's homotopy extension theorem.
\end{abstract}

\section{Introduction}

The classes of absolute retracts $\mathcal{A R}$ and of absolute neighborhood retracts $\mathcal{A N \mathcal { N }}$ (for metrizable spaces) where introduced by K. Borsuk. They play an important role in Homotopy Theory and in Algebraic Topology essentially because, in the category $\mathcal{M}$ of metrizable spaces, $\mathcal{A R}$ is the class of injective objects with respect to closed embeddings, while ANR-spaces are homotopically equivalent to CW-spaces and to polyhedra. Borsuk's theorems characterizing $\mathcal{A R}$ and $\mathcal{A N \mathcal { N }}$ ([3], Thm. 4.2, p. 87) are concerned with closed pairs of metrizable spaces. We generalizes them to pairs $(X, B)$, where $X$ is an arbitrary topological space and $B$ is a $\mathrm{P}$-embedded $([\mathbf{2}])$ zero-set of $X$.

Borsuk's homotopy extension theorem ([3], Thm. 8.1, p. 94) asserts that every closed pair $(X, B)$ of metrizable spaces has the homotopy extension property with respect to the class $\mathcal{A N \mathcal { R }}$. The latest and most remarkable generalization of this theorem is due to Morita $([\mathbf{1 1}])$. He showed that the same result holds for every pair $(X, B)$, where $B$ is a P-embedded, zero-set of $X$. The definition of P-embedded subset used there is different from the previous one but the two are equivalent as shown in $[8]$. We were led to rediscover such a result, for which we present a new and simple proof.

$\mathcal{M}$ is a proreflective subcategory of the category $\mathcal{T}$ of all topological spaces, hence every space can be approximated by an inverse system of metrizable spaces (see Section 1). This fact allows one to extend Borsuk's original proofs from closed pairs in $\mathcal{M}$ to general pairs $(X, B)$ in $\mathcal{T}$, where $B$ is a $\mathrm{P}$-embedded zero-set of $X$.

\section{Preliminaries}

Let $\mathcal{C}$ be a fixed category. In the following we shall work within the category Pro $\mathcal{C}$ of inverse systems in $\mathcal{C}$. We refer to [10] for all details of its definition. We only

Received October 24, 2002, revised June 17, 2003; published on June 30, 2003.

2000 Mathematics Subject Classification: 55P05, 54E35, 54C20, 54C56, 18 A30.

Key words and phrases: continuous pseudometric, metric expansion, proreflection, P-embedding, absolute (neighborhood) retract, homotopy extension property.

(C) 2003, L. Stramaccia. Permission to copy for private use granted. 
recall the following basic facts that will be explicitly used in the sequel :

- an inverse system in $\mathcal{C}$ is a contravariant functor $\mathrm{X}: \Lambda \rightarrow \mathcal{C}$, where $\Lambda=(\Lambda, \leqslant)$ is a directed set, and will be denoted as $\mathrm{X}=\left(X_{\lambda}, x_{\lambda \lambda^{\prime}}, \Lambda\right)$, where $X_{\lambda}=\mathrm{X}(\lambda)$, for every $\lambda \in \Lambda$, and $x_{\lambda \lambda^{\prime}}: X_{\lambda^{\prime}} \rightarrow X_{\lambda}$, where $x_{\lambda \lambda^{\prime}}=\mathrm{X}\left(\lambda \leqslant \lambda^{\prime}\right)$.

- a morphism $\mathrm{p}: X \rightarrow \mathrm{X}$, where $X \in \mathcal{C}$, is simply a cone, i.e. a family $\mathrm{p}=\left\{p_{\lambda}\right.$ : $\left.X \rightarrow X_{\lambda} \mid \lambda \in \Lambda\right\}$ of morphisms of $\mathcal{C}$ having the property that $x_{\lambda \lambda^{\prime}} \cdot p_{\lambda^{\prime}}=p_{\lambda}$, for all $\lambda \leqslant \lambda^{\prime}$.

- if $\mathcal{K}$ is a full subcategory of $\mathcal{C}$ then Pro $\mathcal{K}$ is a full subcategory of ProC. $\mathcal{K}$ is said to be proreflective in $\mathcal{C}$ whenever Pro $\mathcal{K}$ is reflective in ProC. This amounts to saying ([4], [12]) that, for every $X \in \mathcal{C}$, there exists an inverse system $\mathrm{X} \in$ ProK and a morphism $\mathrm{p}: X \rightarrow \mathrm{X}$ in ProC having the following properties :

(1) for every $f: X \rightarrow K, K \in \mathcal{K}$, there exists a $\lambda \in \Lambda$ and a morphism $f_{\lambda}: X_{\lambda} \rightarrow K$, such that $f_{\lambda} \cdot p_{\lambda}=f$.

(2) if $f_{\lambda^{\prime}}: X_{\lambda^{\prime}} \rightarrow K$ has also the property that $f_{\lambda^{\prime}} \cdot p_{\lambda^{\prime}}=f$, then there exists a $\lambda^{\prime \prime} \in \Lambda, \lambda, \lambda^{\prime} \leqslant \lambda^{\prime}$, such that $f_{\lambda} \cdot x_{\lambda \lambda^{\prime \prime}}=f_{\lambda^{\prime}} \cdot x_{\lambda^{\prime} \lambda^{\prime}}$.

If this is the case, then $\mathrm{p}: X \rightarrow \mathrm{X}$ is said to be a $\mathcal{K}$-expansion for $X$. It is clear that a $\mathcal{K}$-expansion for $X$ is uniquely determined up to isomorphisms in ProK

A subcategory $\mathcal{K}$ of the category $\mathcal{T}$ of topological spaces (resp. $\mathcal{T}_{2}$ of Hausdorff spaces) is proreflective if and only if $[\mathbf{6}]$ it is closed under finite products and subspaces (resp. closed subspaces). With this result at hand, one can produce a great number of proreflective subcategories of topological spaces. In particular, the categories $\mathcal{P \mathcal { M }}$ and $\mathcal{M}$ of pseudometrizable and metrizable spaces, respectively, are both proreflective in $\mathcal{T}$. In what follows we discuss these facts.

Let $X=(X, \tau)$ be a topological space. Recall that a pseudometric on $X$ is a map $\lambda: X \times X \rightarrow \mathbb{R}^{+}$having the following properties:

(i) $\lambda(x, x) \geqslant 0$,

(ii) $\lambda(x, y)=\lambda(y, x)$,

(iii) $\lambda(x, y)+\lambda(y, z) \leqslant \lambda(x, z)$,

for all $x, y, z \in X . \lambda$ is said to be continuous $[\mathbf{2}]$ if it is continuous with respect to the product topology on $X \times X$. If $\tau_{\lambda}$ denotes the topology induced on $X$ by the pseudometric $\lambda$, then $\lambda$ will be continuous if and only if $\tau_{\lambda} \subset \tau$ holds.

Let $\Lambda$ be the set of all continuous pseudometrics on $X$, modulo the relation that identifies two pseudometrics whenever they induce the same topology on $X$. $\Lambda$ is directed by the relation $\lambda \leqslant \lambda^{\prime} \Longleftrightarrow \tau_{\lambda} \subset \tau_{\lambda^{\prime}}$.

For every $\lambda \in \Lambda$, let $X_{\lambda}$ denote the metric identification of the pseudometric space $\left(X, \tau_{\lambda}\right)$ while, for $\lambda \leqslant \lambda^{\prime}, x_{\lambda \lambda^{\prime}}: X_{\lambda^{\prime}} \rightarrow X_{\lambda}$ is the map induced on the quotients by the the identity $\left(X, \tau_{\lambda^{\prime}}\right) \rightarrow\left(X, \tau_{\lambda}\right)$, which is continuous. Then $\mathrm{X}=\left(X_{\lambda}, x_{\lambda \lambda^{\prime}}, \Lambda\right)$ is an inverse system in the category $\mathcal{M}$ and the morphism $\mathrm{x}: X \rightarrow \mathrm{X}$ where, for $\lambda \in \Lambda$, $x_{\lambda}: X \rightarrow X_{\lambda}$ is the identity $(X, \tau) \rightarrow\left(X, \tau_{\lambda}\right)$ followed by the identification map, turns out to be a $\mathcal{M}$-expansion for $X[\mathbf{6}],[\mathbf{7}]$. We call it the metrizable expansion of $X$. 
Recall from $[\mathbf{2}]$ that a subset $B$ of a topological space $X$ is said to be $P$-embedded in $X$ if every continuous pseudometric on $B$ can be continuously extended over $X$. An equivalent formulation for this definition is given in [8], Thm. 2.10.

Note that, whenever $B$ is P-embedded in $X$, then the metrizable expansion $\mathrm{b}$ : $B \rightarrow \mathrm{B}$ of $B$ is obtained by restriction from that of $X$. In fact, in such a case every continuous pseudometric on $B$ can be thought of as the restriction of any of its extensions to $X$.

The embedding $i: B \rightarrow X$ induces a level morphism, actually a natural transformation of functors, $\mathrm{i}: \mathrm{B} \rightarrow \mathrm{X}, \quad \mathrm{i}=\left\{i_{\lambda}: B_{\lambda} \rightarrow X_{\lambda} \mid \lambda \in \Lambda\right\}$. That is, for every $\lambda \in \Lambda$, there is a commutative diagram

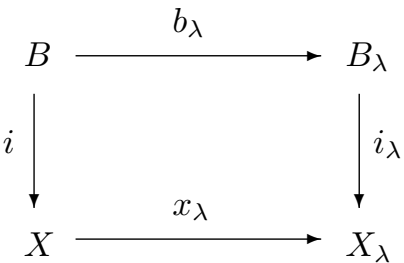

with $i_{\lambda}$ the corresponding inclusion.

Proposition 1.1. Let $X$ be a topological space and let $\mathrm{x}: X \rightarrow \mathrm{X}$ be its metrizable expansion. For a $P$-embedded subset $B$ of $X$ the following properties are equivalent:

(1) $B$ is a zero-set of $X$.

(2) For every $\mu \in \Lambda$, there exists $\lambda \in \Lambda, \lambda \geqslant \mu$, such that $B_{\lambda}$ is closed in $X_{\lambda}$.

Proof. Let $B$ be a P-embedded, zero-set of $X$. There exists a continuous map $f$ : $X \rightarrow \mathbb{R}$ such that $B=f^{-1}(0)$. From the universal property of the metrizable expansion of $X$ one obtains, for some $\eta \in \Lambda$, a map $f_{\eta}: X_{\eta} \rightarrow \mathbb{R}$ such that $f=f_{\eta} \cdot x_{\eta}$. It follows $B=f^{-1}(0)=x_{\eta}^{-1} f_{\eta}^{-1}(0)$, hence $B_{\eta}=x_{\eta}(B)=f_{\eta}^{-1}(0)$. For any $\mu \in \Lambda$, let $\lambda \geqslant \eta$, $\mu$, then $B_{\lambda}=f_{\lambda}^{-1}(0)$, for $f_{\lambda}=f_{\eta} \cdot x_{\eta \lambda}$. Let now $B$ be a P-embedded subset of $X$ for which (2) holds and let $\lambda \in \Lambda$ be any continuous pseudometric on $X$ such that $B_{\lambda}$ is closed in $X_{\lambda}$. Here $B_{\lambda}$ denotes the pseudometrizable subspace of $X_{\lambda}$ defined by $B$. Then $B_{\lambda}$ is a zero-set of $X_{\lambda}$, hence there exists a continuous map $f_{\lambda}: X_{\lambda} \rightarrow \mathbb{R}$ with $B_{\lambda}=f_{\lambda}^{-1}(0)$. It follows that $B=\left(f_{\lambda} \cdot x_{\lambda}\right)^{-1}(0)$.

In the following we shall say that a subset $B$ of a space $X$ is $P_{0}$-embedded if it is P-embedded and satisfies the equivalent conditions of the above proposition.

\section{Examples 1.2.}

1. Every closed subset $B$ of a metrizable space $X$ is $P_{0}$-embedded. This follows from Arens' theorem ([2], 15.4), which states that every closed subset of a metrizable space is P-embedded.

2. The $P_{0}$-embedded subsets of a collectionwise normal space are exactly its zero-sets ([2], 15.7).

3. Every closed, P-embedded, $G_{\delta}$-subset of a normal space is $P_{0}$-embedded.

4. Every closed, P-embedded subset of a perfectly normal $T_{1}$-space is $P_{0}$-embedded.

5. Every $C$-embedded zero-set of a normal, countably paracompact space is $P_{0}$ embedded. 


\section{Results}

The machinery we have given in the previous section allows us to obtain the following generalization of Borsuk's characterization theorems for AR and ANR spaces.

Theorem 2.1. Let $Y$ be a metrizable space. Then

(1) $Y \in \mathcal{A R}$ if and only if, for every pair $(X, B)$ of topological spaces, where $B$ is $P_{0}$-embedded in $X$, every map $f: B \rightarrow Y$ has a continuous extension $\tilde{f}: X \rightarrow Y$.

(2) $Y \in \mathcal{A N \mathcal { R }}$ if and only if, for every pair $(X, B)$ of topological spaces, where $B$ is $P_{0}$-embedded in $X$, every map $f: B \rightarrow Y$ has a continuous extension $\tilde{f}: U \rightarrow Y$, to some neighborhood $U$ of $B$ in $X$.

Proof. (1) Let $Y \in \mathcal{A R}$ and let $f: B \rightarrow Y$ be any map. Since $Y$ is metrizable, by the universal property of the metrizable expansion $\mathrm{b}: B \rightarrow \mathrm{B}$, one can find $\lambda \in \Lambda$ and a map $g_{\lambda}: B_{\lambda} \rightarrow Y$ such that $g_{\lambda} \cdot b_{\lambda}=f$. Since $B$ is $P_{0}$-embedded in $X$, then one can assume that $B_{\lambda}$ is closed in the metrizable space $X_{\lambda}$. Applying now the first theorem of Borsuk to the pair $\left(X_{\lambda}, B_{\lambda}\right)$, there exists a map $h_{\lambda}: X_{\lambda} \rightarrow Y$ which extends $g_{\lambda}$, that is $h_{\lambda} \cdot i_{\lambda}=g_{\lambda}$. Finally, $f=g_{\lambda} \cdot b_{\lambda}=h_{\lambda} \cdot i_{\lambda} \cdot b_{\lambda}=h_{\lambda} \cdot x_{\lambda} \cdot i$, so that $\tilde{f}=h_{\lambda} \cdot x_{\lambda}$ is an extension of $f$. Conversely, let every map $f: B \rightarrow Y$ have an extension $\tilde{f}: X \rightarrow Y$, whenever $B$ is $\mathrm{P}_{0}$-embedded in $X$. Since this holds in particular for every closed pair $(X, B)$ of metrizable spaces, from Borsuk's theorem it follows that $P$ has to be an AR.

(2) The proof of the second part is analogous to that of the first one, unless to take an extension $h_{\lambda}: U_{\lambda} \rightarrow Y$ of $g_{\lambda}$, for $U_{\lambda}$ a neighborhood of $B_{\lambda}$ in $X_{\lambda}$, whose existence is guaranteed by the second Borsuk theorem. Then consider $U=x_{\lambda}^{-1}\left(U_{\lambda}\right)$ to obtain the extension $\tilde{f}=h_{\lambda} \cdot x_{\lambda}: U \rightarrow Y$ of $f$.

Recall that a continuous map $i: B \rightarrow X$ is said to have the homotopy extension property (HEP) with respect to a class $\mathcal{K}$ of topological spaces, whenever the diagram

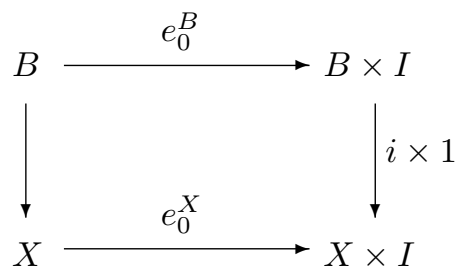

is a weak pushout with respect to $\mathcal{K}$. This means that, for every map $g: X \rightarrow$ $Y, Y \in \mathcal{K}$, and homotopy $F: B \times I \rightarrow Y$ such that $F \cdot e_{0}^{B}=g \cdot i$, there exists a homotopy $G: X \times I \rightarrow Y$ with $G \cdot e_{0}^{X}=g$ and $G \cdot(i \times 1)=F$. Here $e_{0}^{X}: X \rightarrow X \times I$ is the map defined by $e_{0}^{X}(x)=(x, 0)$, for all $x \in X$. In the sequel we will consider the HEP for a pair $(X, B)$ of topological spaces, where $B$ is a subset of $X$ and $i: B \rightarrow X$ is the embedding map.

Theorem 2.2. (The Homotopy Extension Theorem) A pair of topological spaces $(X, B)$ has the homotopy extension property with respect to $\mathcal{A} \mathcal{N} \mathcal{R}$ whenever $B$ is $P_{0}$-embedded in $X$. 
Proof. Given a commutative diagram

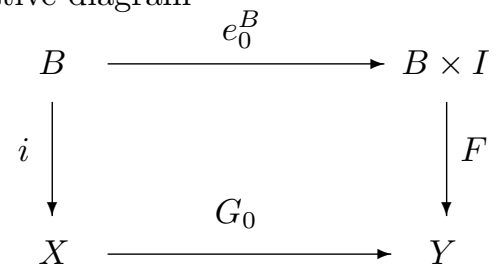

where $Y \in \mathcal{A N \mathcal { R }}$, we have to construct an extension $G: X \times I \rightarrow Y$ of $F$, such that $G \cdot e_{0}^{X}=G_{0}$.

Note that the metrizable expansion of $X \times I$ is given by $\mathrm{x} \times 1: X \times I \rightarrow \mathrm{X} \times I=$ $\left(X_{\lambda} \times I, x_{\lambda \lambda^{\prime}} \times 1, \Lambda\right)$, and the related metrizable expansion of $B \times I$ by b $\times 1: B \times I \rightarrow$ $\mathrm{B} \times I=\left(B_{\lambda} \times I, b_{\lambda \lambda^{\prime}} \times 1, \Lambda\right)$. By the proreflection property, there exists an index $\lambda \in \Lambda$ and a homotopy $F^{\lambda}: B_{\lambda} \times I \rightarrow Y$, such that $F^{\lambda} \cdot\left(b_{\lambda} \times 1\right)=F$. On the other hand, there exists a $\lambda^{\prime} \in \Lambda$ and a map $G_{0}^{\lambda^{\prime}}: X_{\lambda} \rightarrow Y$, such that $G_{0}^{\lambda^{\prime}} \cdot x_{\lambda^{\prime}}=G_{0}$. Since the equality $G_{0}^{\lambda^{\prime}} \cdot i_{\lambda^{\prime}} \cdot b_{\lambda^{\prime}}=F_{\lambda} \cdot e_{0}^{B_{\lambda}} \cdot b_{\lambda}=G_{0} \cdot i$ holds, then there exists a $\lambda^{*} \geqslant \lambda, \lambda^{\prime}$ such that $F_{\lambda} \cdot e_{0}^{B_{\lambda}} \cdot b_{\lambda \lambda^{*}}=G_{0}^{\lambda^{\prime}} \cdot i_{\lambda^{\prime}} \cdot b_{\lambda^{\prime} \lambda^{*}}$. Since $B$ is $P_{0}$-embedded in $X$ we can assume that, for such a $\lambda^{*}$, the corresponding embedding $i_{\lambda^{*}}$ is closed. Let us define $G_{0}^{\lambda^{*}}=G_{0}^{\lambda^{\prime}} \cdot x_{\lambda^{\prime} \lambda^{*}}$ and $F^{\lambda^{*}}=F^{\lambda} \cdot\left(b_{\lambda \lambda^{*}} \times 1\right)$. Then the following diagram is commutative

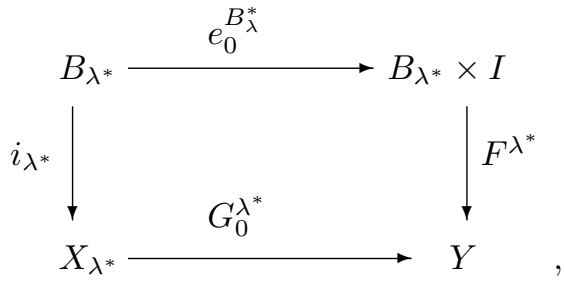

in fact, $F^{\lambda^{*}} \cdot e_{0}^{B_{\lambda^{*}}}=F^{\lambda} \cdot e_{0}^{B_{\lambda}} \cdot b_{\lambda \lambda^{*}}=G_{0}^{\lambda^{\prime}} \cdot i_{\lambda^{\prime}} \cdot b_{\lambda^{\prime} \lambda^{*}}=G_{0}^{\lambda^{\prime}} \cdot x_{\lambda^{\prime} \lambda^{*}} \cdot i_{\lambda^{*}}=G_{0}^{\lambda^{*}} \cdot i_{\lambda^{*}}$. If we apply now Borsuk's homotopy extension theorem to the pair $\left(X_{\lambda^{*}}, B_{\lambda^{*}}\right)$, we get the extension $G^{\lambda^{*}}: X_{\lambda^{*}} \times I \rightarrow Y$ of $F^{\lambda^{*}}$. Finally $G=G^{\lambda^{*}} \cdot\left(x_{\lambda^{*}} \times 1\right)$ turns out to be the extension of $F$ we were searching for.

\section{References}

[1] R.A. Alò - L.I. Sennot, Collectionwise normality and the extension of functions on product spaces, Fund. Math., 76 (1972), 231-243.

[2] R.A. Alò - H.L. Shapiro, Normal topological spaces, C.U.P., 1974.

[3] K. Borsuk, Theory of Retracts, Polish Scientific Publishers, Warsawa 1967.

[4] J.M. Cordier - T. Porter, Shape Theory. Categorical methods of approximation, Ellis Horwood, Chichester 1989.

[5] C.H. Dowker, Homotopy extension theorems, Proc. London Math. Soc. 6(3), (1956), 100-116.

[6] E. Giuli, Relations between reflective subcategories and Shape Theory, Glasnik Mat., 16(36), (1981), 205-210. 
[7] E. Giuli - A. Tozzi, On epidense subcategories of topological categories, Quaest. Math, 6(1983), 197-106.

[8] T. Hoshina, "Extension of mappings II" in Topics in General Topology, K. Morita - J. Nagata eds, 41-80, North-Holland Mathematical Library, 1989.

[9] S.T. Hu, Theory of Retracts, Wayne Univ. Press., 1965.

[10] S. Mardešić - J. Segal, Shape Theory, North Holland, 1982.

[11] K. Morita, On generalizations of Borsuk's homotopy extension theorem, Fund. Math. 88(1975), 1-6.

[12] L. Stramaccia, Reflective subcategories and dense subcategories, Rend. Sem. Mat. Univ. Padova 67(1982), 191-198.

This article may be accessed via WWW at http://www.rmi.acnet.ge/hha/ or by anonymous ftp at

$$
\text { ftp://ftp.rmi.acnet.ge/pub/hha/volumes/2003/n1a9/v5n1a9.(dvi,ps,pdf) }
$$

L. Stramaccia stra@dipmat.unipg.it

Dipartimento di Matematica e Informatica Universitá di Perugia

via Vanvitelli, I-06123 Perugia 